\title{
Normal-Mode Based MUSIC for Bearing Estimation in Shallow Water Using Acoustic Vector Sensors
}

\author{
Weiwei $\mathrm{Ai}^{\mathrm{a}}$, Jinyu Xiong and Xiaoyong Zhang \\ Science and Technology on Blind Signal Processing Laboratory, Chengdu 610041, China \\ aaww_101@163.com
}

\begin{abstract}
Keywords: Acoustic vector sensor array, Normal-Mode based MUSIC, Shallow ocean, Unbiased bearing estimates.
\end{abstract}

\begin{abstract}
To realize unbiased bearing estimates of multiple acoustic sources in a range-independent shallow water, Normal-Mode based MUSIC (NM-MUSIC) method using acoustic vector sensor (AVS) array is proposed in this paper. Comparing to NM-MUSIC method based on scalar array, the method based on AVS array solves the problem of port and starboard ambiguity, and also breaks through the limitation of half wavelength. Meanwhile, the method realizes unbiased bearing estimates, while most of the conventional Direction of Arrival (DOA) methods result in biased bearing estimates in shallow water. Simulation results show that the performance of the new method proposed is better than that of the original method.
\end{abstract}

\section{Introduction}

DOA estimation of underwater targets is an important research content in underwater acoustics. Traditional methods are mostly based on the scalar sensors, which measure only the acoustic pressure. There is an AVS which can not only measure the acoustic pressure, but also get the three orthogonal components of particle velocity at a point. The information contained in the measurements of AVS provides a complete characterization of the acoustic field. The classic methods based on AVS array contain CBF and MUSIC algorithms. But these methods result in biased DOA estimation, because plane-wave propagation is assumed. To solve this problem, Matched field processing (MFP) techniques was proposed by Gong [1], but this method works at the expense of computational complexity because of the three-dimensional search in the bearing-range-depth space. The subspace intersection method given by Lakshmipathi provides high-resolution bearing estimation based on a more appropriate normal mode propagation model [2]. SI alleviates the problems of both bias and computational complexity compared to MFP because of a one-dimensional search of bearing without range and depth. But the robustness of SI is poor because of the QR decomposition. Nagananda [3] extend SI to an array of acoustic vector sensors. Lijie Zhang provided a Normal-Mode based MUSIC (NM-MUSIC) method using scalar sensors, which is adapted from the classical MUSIC method [4]. NM-MUSIC provides unbiased DOA estimates of sources by one dimensional searching without requiring any prior information of source locations. In this paper, we extend NM-MUSIC to an array of acoustic vector sensors, which combines the advantages of NM-MUSIC algorithm and AVS array. Simulations show that the performance of the new method proposed in this paper is better than that of the original method.

\section{AVS array data model based on normal mode propagation model}

The AVS array data model in shallow ocean used in this paper is the same as that described in [3]. A linear AVS array of $N$ sensors at depth $z_{a} \mathrm{~m}$ is taken into account, and the inter-sensor spacing is $d$. There are $J$ narrowband sources of centre frequency $f$, which are located at depth $z_{j} \mathrm{~m}$, ranges $r_{j}$ and bearing $\theta_{j} \quad j=1, \ldots, J$. Bearing is measured with respect to the end-fire direction of the array. AVS can get three orthogonal component of particle velocity $\left(v_{x}, v_{y}, v_{z}\right)$. Among them, $v_{z}$ has little impact on bearing estimates, so we only use the particle velocity $\left(v_{x}, v_{y}\right)$. 
The output of the acoustic vector sensors array could be expressed as

$$
\mathbf{X}(t)=\mathbf{P}(\boldsymbol{\Phi}) \mathbf{S}(t)+\mathbf{n}(t)
$$

where $\mathbf{n}$ is the array noise vector and

$$
\begin{aligned}
& \boldsymbol{\Phi}=[\boldsymbol{\theta}, \mathbf{r}, \mathbf{z}]^{T} \\
& \boldsymbol{\theta}=\left[\theta_{1}, \ldots, \theta_{J}\right]^{T} \\
& \mathbf{r}=\left[r_{1}, \ldots, r_{J}\right]^{T} \\
& \mathbf{z}=\left[z_{1}, \ldots, z_{J}\right]^{T}
\end{aligned}
$$

are the vector of unknown parameters, and

$$
\mathbf{S}(t)=\left[s_{1}(t), s_{2}(t), \cdots, s_{J}(t)\right]^{T}
$$

is the source signal vector, and $\mathbf{P}(\boldsymbol{\Phi})$ is the matrix defined as

$$
\mathbf{P}(\boldsymbol{\Phi})=\mathbf{A}(\boldsymbol{\theta}) \mathbf{B}(\mathbf{r}, \mathbf{z})
$$

and

$$
\begin{aligned}
& \boldsymbol{A}(\boldsymbol{\theta})=\left[\boldsymbol{A}\left(\theta_{1}\right), \ldots, \boldsymbol{A}\left(\theta_{J}\right)\right] \\
& \boldsymbol{B}(\boldsymbol{r}, \boldsymbol{z})=\operatorname{diag}\left(\boldsymbol{b}\left(r_{1}, z_{1}\right), \ldots, \boldsymbol{b}\left(r_{J}, z_{J}\right)\right) \\
& \mathbf{A}\left(\theta_{j}\right)=\left[\mathbf{a}_{1}\left(\theta_{j}\right), \mathbf{a}_{2}\left(\theta_{j}\right), \cdots, \mathbf{a}_{M}\left(\theta_{j}\right)\right] \\
& \mathbf{b}\left(r_{j}, z_{j}\right)=\left[b_{1}\left(r_{j}, z_{j}\right), \ldots, b_{M}\left(r_{j}, z_{j}\right)\right] \\
& b_{m}\left(r_{j}, z_{j}\right)=B_{0} \psi_{m}\left(z_{a}\right) \psi_{m}\left(z_{j}\right)\left[\frac{e^{i k_{m} r_{j}-\delta_{m} r_{j}}}{\sqrt{k_{m} r_{j}}}\right]
\end{aligned}
$$

In these formulas, $M$ is the total number of the normal modes, and $B_{0}$ is a complex quantity independent of $r_{j}, z_{j}, \theta_{j}, z_{a}$. For acoustic vector sensors array,

$$
a_{m}\left(\theta_{j}\right)=\left[1, e^{j k_{m} d \cos \theta_{j}}, \cdots, e^{j(N-1) k_{m} d \cos \theta_{j}}\right]^{T} \otimes\left[1, \frac{k_{m} \cos \left(\theta_{j}\right)}{k}, \frac{k_{m} \sin \left(\theta_{j}\right)}{k}\right]^{T}
$$

$\psi_{m}(z)$ and $k_{m}$ are the eigenfunction and wavenumber of the $m$ th normal mode.

Considering that the sound sources $s_{j}(t)$ are uncorrelated white Gaussian noise with variances $\sigma_{j}^{2}$, and the sound sources $\mathbf{S}(t)$ are uncorrelated with the noise $\mathbf{n}(t)$, and $\mathbf{n}(t)$ is uncorrelated white Gaussian noise with variances $\sigma_{n}^{2}$.

The correlation matrix of $\mathbf{X}(t)$ is defined as

$\mathbf{R}=E\left[\mathbf{X}(t) \mathbf{X}(t)^{\mathrm{H}}\right]$

where the notation $E[\bullet]$ indicates expectation operation, $[\cdot]^{\mathrm{H}}$ is Hermit Transpose.

In practical applications, we use the following formula to estimate the true correlation matrix

$\hat{\mathbf{R}}=\frac{1}{L} \sum_{t=1}^{L}\left[\mathbf{X}(t) \mathbf{X}(t)^{\mathrm{H}}\right]$

where $L$ is the number of snapshots.

\section{NM-MUSIC based on AVS Array}

The bearing estimation method presented in this section is an extended version of NM-MUSIC for a scalar sensor array [4]. According to equation (1) and (7), we can get

$\mathbf{X}=\mathbf{A} \boldsymbol{\Gamma}+\mathbf{n}$

where $\boldsymbol{\Gamma}=\mathbf{B S}$ is independent of source azimuth.

Then, equation (14) can be expressed as

$$
\mathbf{R}=\mathbf{A} E\left[\boldsymbol{\Gamma} \boldsymbol{\Gamma}^{\mathrm{H}}\right] \mathbf{A}^{\mathrm{H}}+E\left[\mathbf{n} \mathbf{n}^{\mathrm{H}}\right]
$$


Considering the spatially white noise with a power of $\sigma_{n}^{2}$, we have

$$
\mathbf{R}=\mathbf{A} \mathbf{Q} \mathbf{A}^{\mathrm{H}}+\sigma_{n}^{2} \mathbf{I}_{3 N}
$$

where $\mathbf{Q}=E\left[\mathbf{\Gamma} \boldsymbol{\Gamma}^{H}\right]$ is a positive definite matrix.

If $J M<3 N$ satisfies, the $\mathbf{A} \mathbf{Q} \mathbf{A}^{\mathrm{H}}$ would be singular. That is

$$
\left|\mathbf{A Q A}^{\mathrm{H}}\right|=\left|\mathbf{R}-\sigma_{n}^{2} \mathbf{I}\right|=0
$$

which means that $\sigma_{n}^{2}$ is an eigenvalue of $\mathbf{R}$.

Suppose $\mathbf{R}$ has $3 N$ eigenvalues eigenvalues $\lambda_{1}, \cdots, \lambda_{3 N}$ and $3 N$ corresponding eigenvectors $\mathbf{e}_{1}, \cdots, \mathbf{e}_{3 N}$ which satisfy

$\mathbf{R e}_{i}=\lambda_{i} \mathbf{e}_{i}$

In the view of MUSIC algorithm, we have

$$
\mathbf{R}=\mathbf{U}_{s} \Sigma_{s} \mathbf{U}_{s}^{\mathrm{H}}+\mathbf{U}_{n} \Sigma_{n} \mathbf{U}_{n}^{\mathrm{H}}
$$

where $\Sigma_{s}=\operatorname{diag}\left\{\lambda_{1}, \cdots, \lambda_{J}\right\} \quad$ and $\quad \Sigma_{n}=\operatorname{diag}\left\{\lambda_{J+1}, \cdots, \lambda_{3 N}\right\}$. Ideally, $\lambda_{J+1}=\lambda_{J+2}=\cdots=\lambda_{3 N}=\sigma_{n}^{2}$ $\mathbf{U}_{s}=\left[\mathbf{e}_{1}, \cdots, \mathbf{e}_{J}\right]$ is the signal space, and $\mathbf{U}_{n}=\left[\mathbf{e}_{J+1}, \cdots, \mathbf{e}_{3 N}\right]$ is the noise space.

According to equation (18), we can get

$\mathbf{B P B}^{H} \mathbf{e}_{i}=\left(\lambda_{i}-\sigma_{n}^{2}\right) \mathbf{e}_{i}, i=1, \cdots, 3 N$

When $\lambda_{k}=\sigma_{n}^{2}$, then $\mathbf{A Q A} \mathbf{A}^{\mathrm{H}} \mathbf{e}_{k}=0$. Since $\mathbf{P}$ is a positive definite matrix, so we have $\mathbf{A e}_{k}=0$. That means the eigenvector $\mathbf{e}_{k}$ is perpendicular to the column space of $\mathbf{A}(\theta)$. So $\mathbf{U}_{n}$ is perpendicular to the column space of $\mathbf{A}(\theta)$. The distance from $\mathbf{U}_{n}$ to $\mathbf{A}(\theta)$ is defined as $\left\|\mathbf{A}(\theta)^{\mathrm{H}} \mathbf{U}_{n} \mathbf{U}_{n}^{\mathrm{H}} \mathbf{A}(\theta)\right\|_{F}$, which is close to 0 in the actual case, and $\left\|_{\bullet}\right\|_{F}$ denotes Frobenius norm. So when we do one dimensional searching for $\mathbf{A}(\theta)$ along $\theta$, the norm is close to zero if $\theta \in\left\{\theta_{1}, \theta_{2}, \cdots, \theta_{J}\right\}$, and $\theta_{j}$ is the direction of the targets. Namely

$$
\theta_{\text {AVS-NM-MUSIC }}=\arg \min _{\theta}\left\|\mathbf{A}(\theta)^{\mathrm{H}} \mathbf{U}_{n} \mathbf{U}_{n}^{\mathrm{H}} \mathbf{A}(\theta)\right\|_{F}
$$

Therefore, the spatial spectrum estimate of NM-MUSIC based AVS array is

$$
P_{\text {AVS-NM-MUSIC }}(\theta)=\frac{1}{\left\|\mathbf{A}(\theta)^{\mathrm{H}} \mathbf{U}_{n} \mathbf{U}_{n}^{\mathrm{H}} \mathbf{A}(\theta)\right\|_{F}}
$$

Calculating steps of NM-MUSIC based AVS array are as follows:

(1) To estimate the array output covariance matrix $\hat{\mathbf{R}}$ as equation (15);

(2) Construct the noise space $\mathbf{U}_{n}$ through eigen decomposition of $\hat{\mathbf{R}}$;

(3) To calculate $P_{\text {AVS-NM-MUSIC }}(\theta)$ by equation (24);

(4) Find the highest $J$ peaks of $P_{A V S-N M-M U S I C}(\theta)$.

The $J$ azimuth angles corresponding to these peaks of $P_{A V S-N M-M U S I C}(\theta)$ are the DOA estimates of the $J$ targets.

The NM-MUSIC for the AVS array has the same form as that for the scalar sensor array. But there are some significant differences in the constraints on the array parameters. For a scalar sensor array of $N$ elements placed in a channel that supports $M$ normal modes, the NM-MUSIC method works out only when $J M<N$. In the case of an AVS array, the condition is satisfied if $J M<3 N$, namely $J<3 N / M$. So when the number of array elements is definite, the AVS array can distinguish more targets than a scalar array. For an AVS array, the vector $a_{m}\left(\theta_{j}\right)$ are phase shifted not only because of the time delay between the array elements, but also the inherent phase shift between the acoustic pressure and the 
three orthogonal components of particle velocity. It leads to the elimination of intersensor spacing $d$ and port and starboard ambiguity.

\section{Simulations}

We consider the Pekeris Ocean model as [3]. The ocean model is shown in Fig.1. The channel parameters are assumed to be the following: water depth $H=75 \mathrm{~m}$, sound speed in water $c=1500 \mathrm{~m} / \mathrm{s}$, sound speed in bottom $c_{b}=1700 \mathrm{~m} / \mathrm{s}$, density of water $\rho=1 \mathrm{~g} / \mathrm{cm}^{3}$, density of bottom $\rho_{b}=1.5 \mathrm{~g} / \mathrm{cm}^{3}$, attenuation in bottom $\delta=0.5 \mathrm{~dB} / \lambda$ where $\lambda=c / f$, and $f=100 \mathrm{~Hz}$. All the sources are located at ranges $r=5000 \mathrm{~m}$ and depths $z_{j}=37.5 \mathrm{~m}$. The acoustic vector sensors array are located at depths $z=5 \mathrm{~m}$, and the inter-sensor spacing $d=\lambda / 2$, the number of sensors $N=20$. All the results have been obtained by averaging over 200 simulations, and the snapshots is $L=350$. The signal-to-noise ratio for the source is defined as [3].

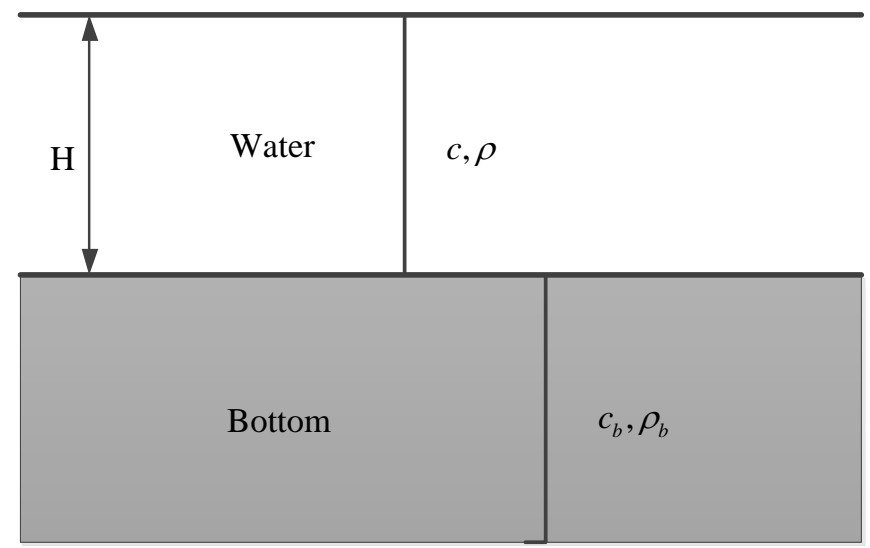

Fig. 1 Ocean model

First, in Fig. 2, we present the pseudo-spectrum performance of the NM-MUSIC, conventional MUSIC and CBF algorithm based on AVS array. Three sources is set at $30^{\circ}, 60^{\circ}$ and $120^{\circ}$ with respect to the array end-fire direction with SNR=10dB. It is shown that: (1) NM-MUSIC based AVS array provides unbiased DOA estimates under the multimode acoustic propagation, while the conventional MUSIC and CBF result in biased DOA estimates; (2) NM-MUSIC based AVS array achieves high-resolution DOA estimates, while the main lobe width of conventional MUSIC and CBF is much wider than NM-MUSIC.

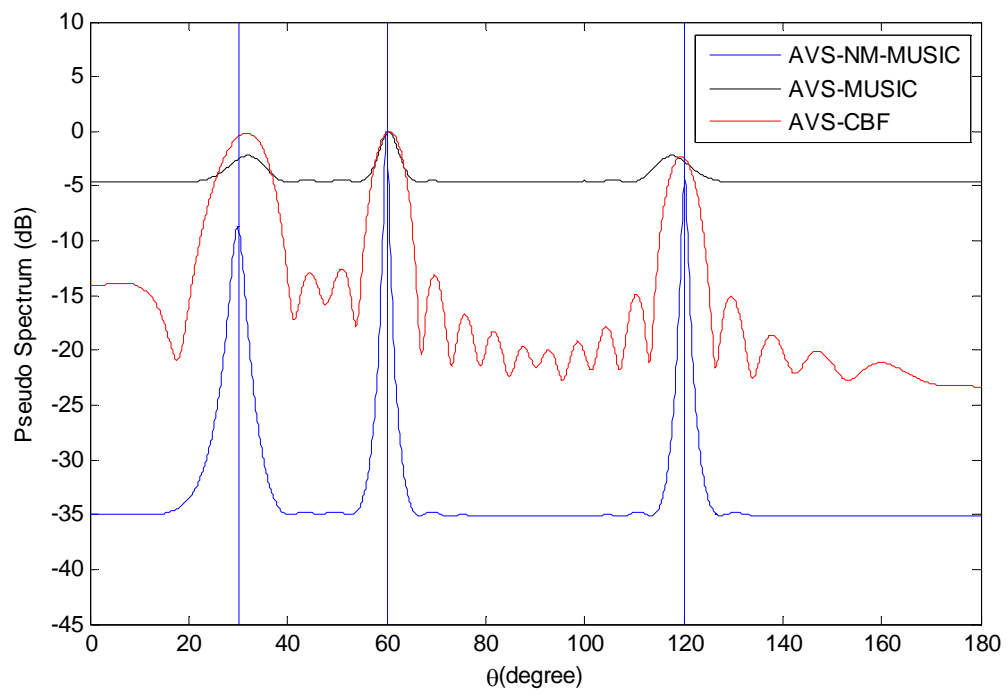

Fig. 2 Pseudo-spectrum of NM-MUSIC, conventional MUSIC and CBF based on AVS array

Fig. 3 shows the DOA estimation bias of NM-MUSIC and conventional MUSIC respectively as the signal incident bearing increase from $10^{\circ}$ to $80^{\circ}$ at $\mathrm{SNR}=10 \mathrm{~dB}$. The direction $0^{\circ}$ is the array end- 
fire direction. It is shown that as the incident bearing increase, the bias of incident bearing estimates by MUSIC and NM-MUSIC decreases, but NM-MUSIC based AVS array provides smaller bias of bearing estimates than MUSIC based AVS array.

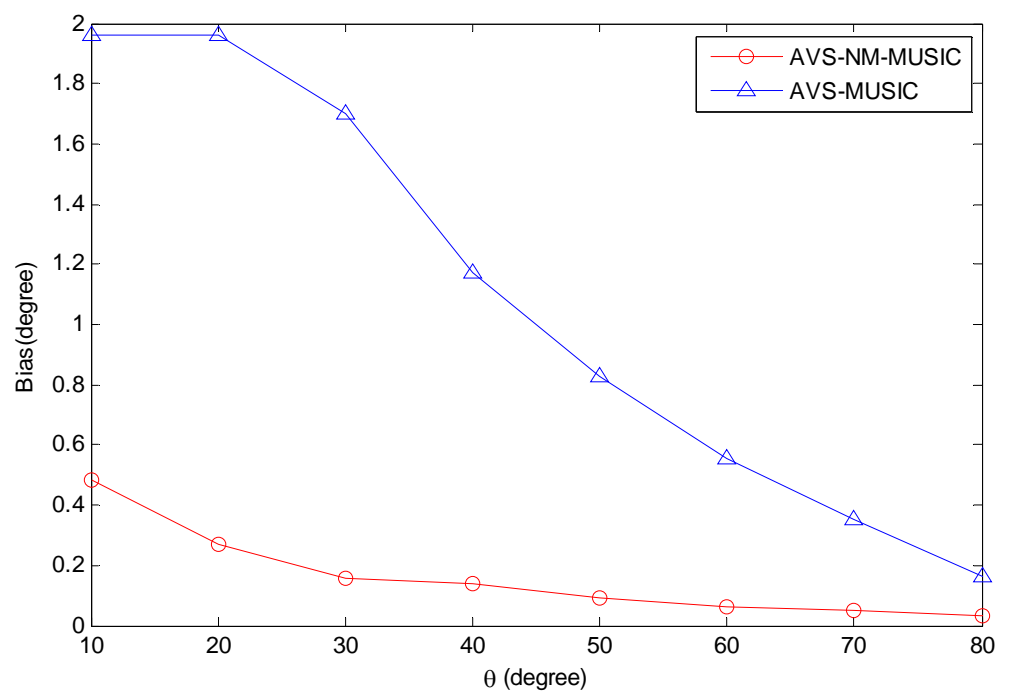

Fig. 3 The bias of NM-MUSIC and MUSIC based AVS array at different incident angles

Fig. 4 gives the pseudo spectrum of NM-MUSIC based AVS array when there are two sources at $30^{\circ}$ and $60^{\circ}$. It is shown that NM-MUSIC based AVS array suppresses the peaks at $-30^{\circ}$ and $-60^{\circ}$ effectively, and achieves accurate DOA estimates without port and starboard ambiguity.

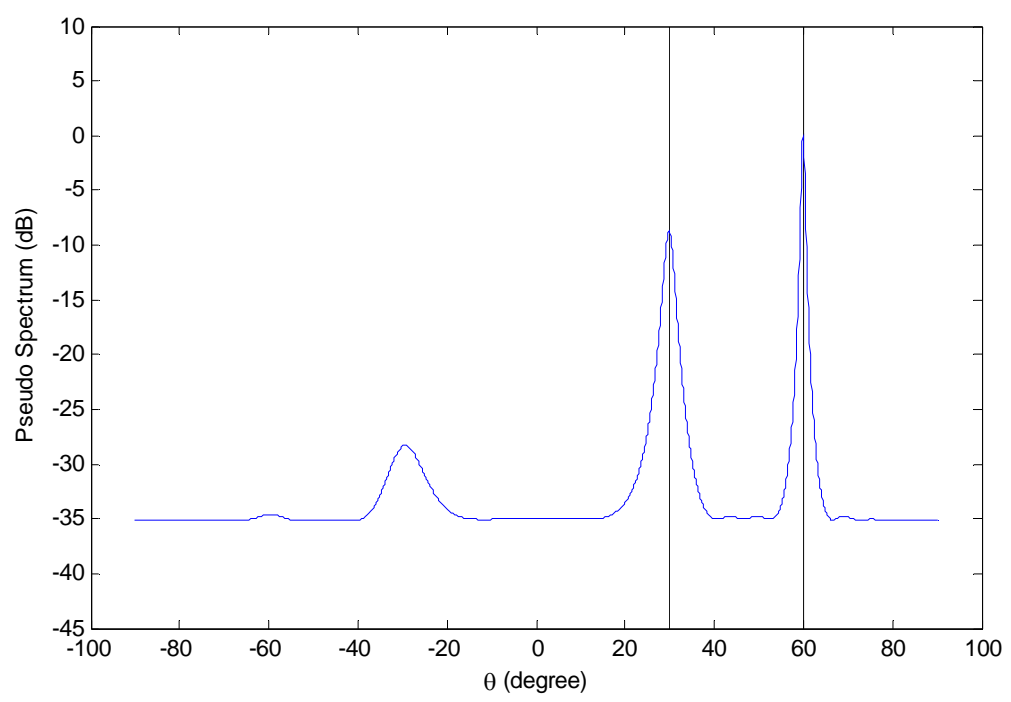

Fig. 4 Pseudo spectrum of NM-MUSIC based AVS array when there are two sources at $30^{\circ}$ and $60^{\circ}$.

Fig. 5 shows the pseudo spectrum of NM-MUSIC based AVS array when there are two sources at $60^{\circ}$ and $62^{\circ}$,inter-sensor spacing $d=1.5 \lambda$. It is shown that NM-MUSIC based AVS array can resolve and localize two sources at $60^{\circ}$ and $62^{\circ}$ with low side-lobes when $d=1.5 \lambda$, since an AVS array works out without the limitations of inter-sensor. 


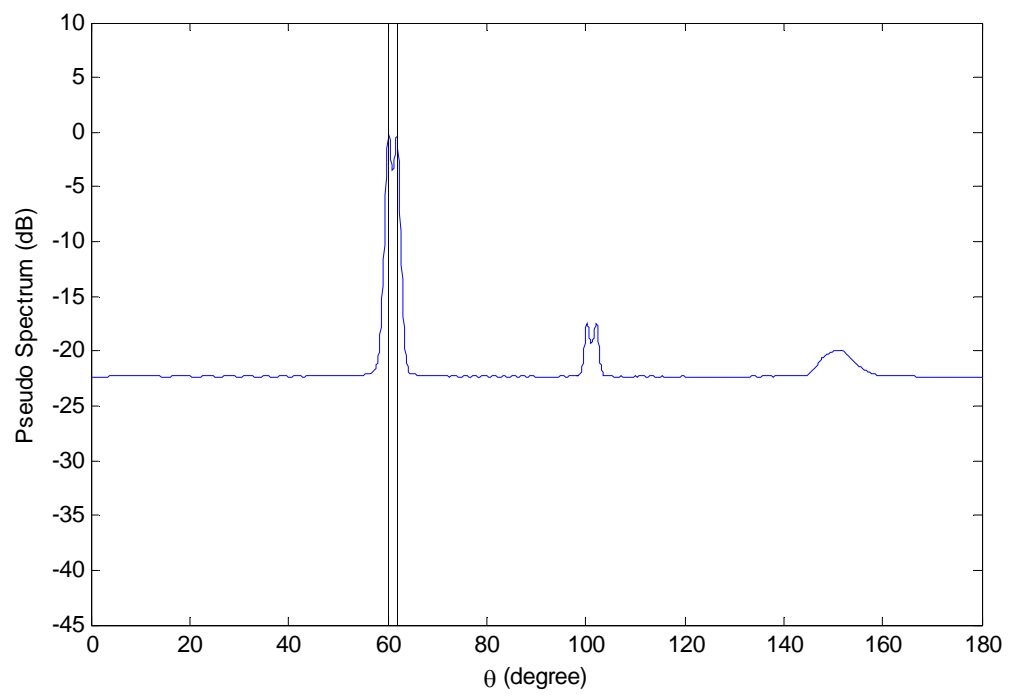

Fig. 5 Pseudo spectrum of NM-MUSIC based AVS array when there are two sources at $60^{\circ}$ and $62^{\circ}$. Inter-sensor spacing $d=1.5 \lambda$.

\section{Conclusion}

In this paper, we have extended NM-MUSIC method to an AVS array in shallow water. The bearing are estimated using a one-dimensional search algorithm without the prior knowledge of source ranges and depths. NM-MUSIC based AVS array achieves unbiased DOA estimates in shallow water by replacing the invalid plane-wave propagation model by a more appropriate normal mode propagation model. NM-MUSIC based AVS array provides smaller bias of bearing estimates than MUSIC based AVS array at different incident bearing. And in the method of NM-MUSIC based AVS array, the inter-sensor spacing does not need to be limited within half-wavelength, and highresolution bearing estimates is realized without port and starboard ambiguity.

\section{References}

[1]. Zaixiao Gong, Jing Lin, Lianghao Guo. The effect of acoustic waves' phase speed on preciseness of DOA estimation in shallow water. Acta Acustica. Vol. 27 (2002), p. 492-496.

[2]. S. Lakshmipathi, and G. V. Anand. Subspace intersection method of high-resolution bearing estimation in shallow ocean. Signal Processing. Vol. 84 (2004), p. 1367-1384.

[3]. K. G. Nagananda, and G. V. Anand. Subspace intersection method of high-resolution bearing estimation in shallow ocean using acoustic vector sensors. Signal Processing. Vol. 84 (2010), p. 1367-1384.

[4]. Lijie Zhang, Jianguo Huang, and Qunfei Zhang. Normal-Mode based MUSIC for bearing estimation in shallow water. Sensor Array and Multichannel Signal Processing Workshop. Darmstadt, Germany, 2008, p. 91-94. 